\title{
Increasing of Acromegaly Prevalence in Guayaquil, Ecuador: 2000-2019
}

\author{
Segura Mestanza Abigail ${ }^{1,3}$, López Gavilanez Enrique ${ }^{1,2 *}$, Cedeño German Roberto ${ }^{1,3}$, Bautista

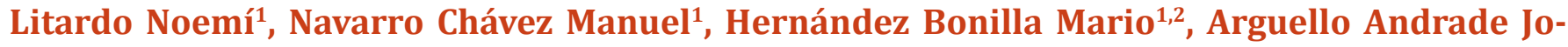 \\ hanna $^{1,3}$, Valdivieso Jara Judith ${ }^{1,3}$, López Estrella Camilo ${ }^{1,4}$ and Solorzano Romero Narcisa ${ }^{1}$ \\ ${ }^{1}$ AECE Research Group, from of the Association of Clinical Endocrinologists of Ecuador
}

${ }^{2}$ Servicio de Endocrinología. Hospital Docente Policía Nacional Guayaquil No. 2. Guayaquil, Ecuador

${ }^{3}$ Servicio de Endocrinología. Hospital Abel Gilbert Pontón. Guayaquil, Ecuador

${ }^{4}$ Servicio de Endocrinología. Hospital Teodoro Maldonado Carbo. Guayaquil, Ecuador

*Corresponding author: Enrique López Gavilanez, Servicio de Endocrinología. Hospital Docente Policía Nacional Guayaquil No.2. Avenida de las Américas S/N y E.Noboa. Guayaquil, Guayas, Ecuador, Email: enrique_lopezg57@hotmail.com

To Cite This Article: Segura Mestanza Abigail, López Gavilanez Enrique, Cedeño German Roberto, Increasing of Acromegaly Prevalence in Guayaquil, Ecuador: 2000-2019. 2020 - 10(4). AJBSR.MS.ID.001535. DOI: 10.34297/AJBSR.2020.10.001535.

Received: 制 September 28, 2020; Published: 眥 October 12, 2020

\begin{abstract}
Background: Reliable and up-to-date information on the epidemiology of acromegaly in Latin American countries remains to be lacking.

Aims: This study aims to update the epidemiological data on acromegaly in Guayaquil city, Ecuador.

Methods: Prospective and retrospective data of patients with acromegaly were collected from the five public health system hospitals in Guayaquil City, Ecuador, from January 2000 to December 2019. Age at diagnosis, baseline growth hormone levels, growth hormone after oral glucose overload, and serum insulin-like growth factor-1 concentrations were recorded. We determined the rate of prevalence of acromegaly using the 2010 population census information and the projected Ecuadorian population by calendar year and canton for the period from 2000 to 2019.

Results: A total of 132 cases of acromegaly were reported, of which 77 are women (58 \%) and 55 men (42 \%) (Female-to-male ratio, 1.4:1). The overall mean age at diagnosis was 46 years (SD, 16 y). The mean age in women was determined to be 46.9 years (SD, 16.3 y), while for the men, it was 45.1 years (SD, $15.8 \mathrm{y}$ ). Our findings show that the prevalence of acromegaly is 49 cases per million inhabitants.
\end{abstract}

Conclusions: The prevalence of acromegaly in Guayaquil, Ecuador are higher than what was previously reported and are closer to those described in the international literature.

Keywords: Acromegaly; Gigantism; Epidemiology; Prevalence; Guayaquil; Ecuador

Abbreviations: GH Growth Hormone; IGF-1 Insulin-like Growth Factor 1

\section{Introduction}

Acromegaly is known to be an orphan disease caused by the hypersecretion of growth hormone (GH) [1]. In order to generate reliable epidemiological data on acromegaly, large population studies are required [1]. Acromegaly has been determined to affect both men and women; it often has late diagnosis, at a mean age of 40 to 50 years, because of its insidious onset and slow progression [1].
The epidemiology of this disease has been studied extensively in developed countries, and significant changes have been observed over time in different regions and countries. Globally, the estimated prevalence of acromegaly is at 28 to 137 cases per million inhabitants and the estimated annual incidence at 2 to 11 new cases per million inhabitants [2-4]. In Europe, the prevalence varies from 4 to 10 cases per million inhabitants [4]. Recent studies 
have revealed that both Iceland and Malta have recorded increasing prevalence in the past 5 years ( $>13$ cases per 100,000 inhabitants) $[5,6]$; meanwhile, basing on the national registries, Sweden, Spain, and Denmark have reported a lower prevalence, with 36-39 cases per million inhabitants $[3,7]$.

Incidence studies published before 2000 have reported a worldwide annual incidence consistently less than five cases per million inhabitants $[2,8]$. However, the global annual incidence reported in more recent studies is found to be much higher, ranging from 2 to 11 cases per million inhabitants $[5,6,9,10]$.

Data from cancer registries tend to sub-report benign tumors, including pituitary adenomas. In addition, data obtained from isolated tertiary reference centers worldwide are deemed affected by selection bias and wide variations in reference patterns [8]. Studies based on national acromegaly registries can provide broader and more longitudinal snapshots of acromegaly in each country $[1,2,8]$. Most of these studies come from European countries and the United States [8].

There are no national acromegaly registries in Latin American countries, except for Mexico. Among the four Latin American countries in which the epidemiology of acromegaly has been studied (Mexico [11], Brazil [12], Argentina [13], and Ecuador [14]), the prevalence of this disease was determined to range from 18 to 365 cases per million inhabitants. It should be noted that the population, design of the studies, and methodology used (not based on the national population, small sample size, and cohort of pituitary tumors) differ greatly between these countries. The incidence in these countries ranges from 1.3 to 9.2 cases per million inhabitants, although with similar methodological drawbacks $[13,14]$. In 2016, Lopez et al [14], described the epidemiology of acromegaly in Guayaquil city, finding a prevalence of 18.7 cases per million inhabitants and an incidence of 1.3 cases per million peopleyears. In this current study, we sought to update the epidemiology of acromegaly based on the information recorded in the past 20 years.

\section{Materials and Methods}

This is a multicenter, longitudinal, prospective, and retrospective study. Data were collected from patient visits to the outpatient clinics of endocrinology departments of the five participating hospitals during the study period (2000-2019). Information such as age, sex, age at diagnosis, biochemical tests, pituitary imaging studies, were recorded. A biochemical diagnosis of acromegaly can be established when the nadir GH after a 75 g glucose load was greater than $1 \mathrm{ng} / \mathrm{mL}$ and there was also an elevated IGF-1 level for age and sex. To measure the prevalence of this disease, information from the 2010 population census provided by the National Statistics and Census Institute of Ecuador [15] and the projected Ecuadorian population by calendar year and canton for the period from 2000 to 2019 were used [16].In 2019, Guayaquil had a population of $2,698,077$ people, representing $24.5 \%$ of the total population of Ecuador (51\% women) [16].

\section{Results}

Between January 2000 and December 2019, a total of 132 patients were diagnosed with acromegaly in Guayaquil, 77 of whom are women (58\%) and 55 men (42\%). The female-to-male ratio was found to be 1.4:1. Patients were categorized by age group at the time of diagnosis, by decade $(<20->80)$, and by sex (Figure1). Most patients aged between 39 and 59 years at the time of diagnosis. The overall mean age at diagnosis was 46 years (SD, 16 y), with 46.9 years (SD, $16.3 \mathrm{y}$ ) in women and 45.1 years (SD, $15.8 \mathrm{y}$ ) in men. Ten patients were found to be younger than 20 years old at the time of diagnosis, and one aged more than 80 years old. There were more women than men in all age groups except for the group of patients between 50 and 59 years old, but there were no women at either extremes of age (Figure 1).Within the 20 -year study period, the estimated prevalence of acromegaly was determined to be 48.92 cases per million population (Table 1). Comparing the period from 2000 to 2014 with that from 2000 to 2019 , its prevalence was seen to increase 2.6 times. The maximum increase in prevalence was determined in the last 5-year period (2015-2019), during which it rose to 31 cases per million inhabitants (Table 1).

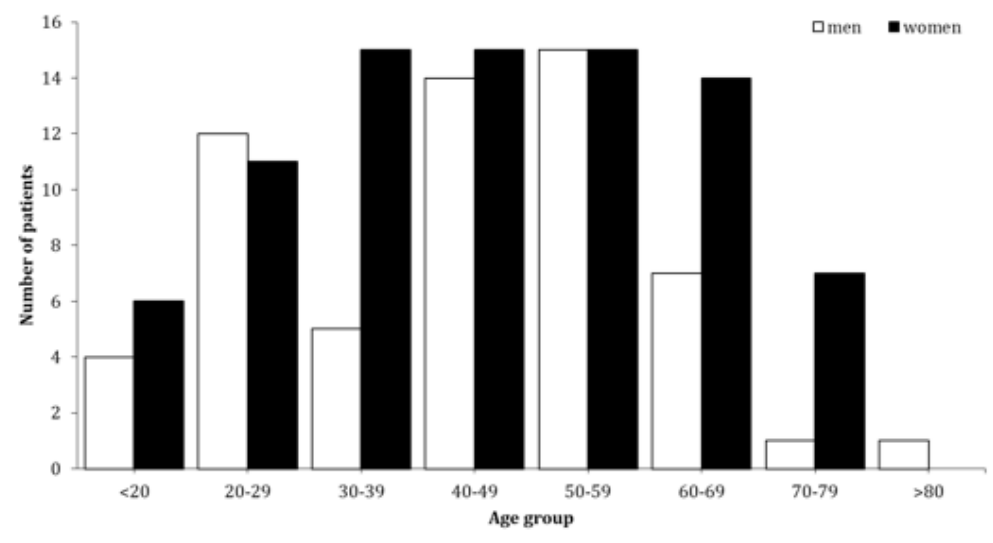

Figure 1: Acromegaly cases by age group in Guayaquil, Ecuador. 2000-2019. 
Table 1: The Prevalence of acromegaly in Guayaquil, Ecuador 2000-2019.

\begin{tabular}{|c|c|c|c|c|c|}
\hline & No. of patients & Mean age at diagnosis (SD) & Population of Guayaquil & Prevalence* & F:M ratio \\
\hline $2000-2014$ & 48 & $47.3(16.8)$ & 2560505 & 18.7 \\
\hline $2015-2019$ & 84 & $46.7(15.7)$ & 2698077 & 31.13 \\
\hline $2000-2019$ & 132 & $46.1(16.1)$ & 2698077 & 48.9 \\
\hline
\end{tabular}

(SD) standard deviation; * per million population

\section{Discussion}

Globally, there are differences in terms of the prevalence of acromegaly between regions and countries. In Europe, before 2000 , the overall prevalence of acromegaly was less than 7 cases per 100,000 inhabitants [2,7]. More recently, in the past 5 years, its prevalence has increased to more than 13 cases per 100,000 inhabitants $[5,6,9]$, as per the studies conducted in the populations of Iceland and Malta. This may be attributed to the improvements in the diagnostic methods, which aim to likely reduce under diagnosis. It is also possible that the diagnosis may have improved as a result of the availability of large national databases in recent population studies [8]. Increased prevalence may also be a result of increased patient survival, owing to better surgical techniques, post-surgical follow-up, and medical treatment [8]. However, the same case was not reported in Spain, Denmark, and Sweden, which all recorded a lower prevalence of acromegaly (3.6-3.9 cases per 100,000 inhabitants) [3,7].

In Latin American countries, the prevalence of acromegaly was found to range from 18 to 365 cases per million inhabitants. Its prevalence is 18 cases per million inhabitants in Mexico [11], 365 cases per million inhabitants in Brazil [12], 140 cases per million inhabitants in Argentina [13], and 18.7 per million inhabitants in Ecuador [14] The prevalence reported in this study (48.9 cases per million inhabitants) is 2.6 times higher than that previously reported in our city [14] and is similar in range to those described in the international literature. This could indicate a significant decrease in under diagnosis. However, this numbers might still be small as not all cases attended in the hospitals of the city's private health system are accounted.

In the literature review, patients with acromegaly are generally diagnosed in the fourth to fifth decade of life $[2,3,8]$. Like in other countries, our patients were diagnosed at an average age of 46 years. Only a small number of these patients (10 before 20 years; 1 after 80 years) were diagnosed at extreme ages, representing 8 $\%$ of the sample. It is important to note that epidemiological data regarding onset of acromegaly (or gigantism) at a younger age are scarce, owing to the rarity of this disease. It has been estimated that only $2.4 \%$ to $22.24 \%$ of patients with this disease aged between 0 and 19 years old [17]. Most studies have shown a predominance of the disease in women, ranging from $51 \%$ to $61 \%$ [2,7]; this is in contrast to some studies reporting no differences in sex prevalence
(51\% vs $49 \%$ ) [18].

In a previous study, we have described the predominance of acromegaly in women (64.5\%), with a female-to-male ratio of 1.8:1. This finding has been confirmed by current study (58\%), showing a female-to-male ratio of $1.4: 1$, which is also in line with what is described in the international literature $[2,8]$. In a cross-sectional study conducted in Quito City, Ecuador, Acosta et al. reported 45 acromegaly cases, where majority (56\%) are women and $44 \%$ are men. Unfortunately, however, the prevalence and incidence rates were not calculated for the population of Quito [19].

Studies that are based on national registries of acromegaly can provide broad and longitudinal descriptions of the epidemiology of the disease in each country. Most of these studies have been conducted in European countries and the United States [7,9,18,20]. In Latin America, only Mexico has a national acromegaly registry, which published its information in 2016 [11].

We should point out the following strengths and weaknesses of this present work. First, this study was conducted in the most populous city in Ecuador (24.5\% of the total population) [15] and in the five hospitals of the public health system that are considered as referral centers for patients throughout the city. We believe that this would provide us with a more realistic view of the epidemiology of acromegaly in our city. Second, hospitals and clinics in the city's private health system did not participate in this study, which could have limited the inclusion of more cases. However, in Guayaquil, more than $90 \%$ of patients with acromegaly are treated in hospitals within the public health system, so our coverage would be almost complete. Third, the sample size of cases with acromegaly is small, making it impossible to extrapolate the results to the entire population of Ecuador.

\section{Conclusions}

With the aim to update the epidemiology of acromegaly in Guayaquil, we observed an increasing trend in prevalence rate, compared with previous reports. The sub-diagnosis and subregistration of cases that we described previously have been reduced.

Having accurate and up-to-date epidemiological data on acromegaly is essential to describe disease patterns, to assess the impact of the disease and its comorbidities on patients, and to examine the burden of acromegaly in the healthcare system. 


\section{Acknowledgements}

The Authors thank ENAGO for its support professional academic English proofreading.

\section{Disclosure}

No potential conflict of interest relevant to this article was reported.

\section{References}

1. Melmed S (2017) Chapter 15 - Acromegaly. The Pituitary (Fourth Edition) pp. 423-466.

2. Colao A, Grasso LFS, Giustina A, Mel med S, Chanson P, et al. (2019) Acromegaly. Nat Rev Dis Primers 5(1): 20.

3. Dal J, Feldt Rasmussen U, Andersen M, Kristensen L, Laurberg P, et al. (2016) Acromegaly incidence, prevalence, complications and long-term prognosis: a nationwide cohort study. Eur J Endocrinol 175(3): 181-190.

4. Orphanet (2020) rare diseases. Acromegaly.

5. Agustsson, TT, Baldvinsdottir, Jonasson JG, Olafsdottir E, Steinthorsdottir $\mathrm{V}$, et al. (2015) The epidemiology of pituitary adenomas in Iceland, 1955-2012: a nationwide population-based study. Eur J Endocrinol 173(5): 655-664.

6. Gruppetta M, Mercieca C, Vassallo J (2013) Prevalence and incidence of pituitary adenomas: a population based study in Malta. Pituitary 16(4): 545-553.

7. Mestron A, Webb SM, Astorga R, Benito P, Catala M, et al. (2004) Epidemiology, clinical characteristics, outcome, morbidity and mortality in acromegaly based on the Spanish Acromegaly Registry (Registro Español de Acromegalia, REA). Eur J Endocrinol 151(4): 439-446.

8. Lavrentaki A, Paluzzi A, Wass JA, Karavitaki N (2017) Epidemiology of acromegaly: review of population studies. Pituitary 20(1): 4-9.

9. Hoskuldsdottir GT, Fjalldal SB, Sigurjonsdottir HA. (2015) The incidence and prevalence of acromegaly, a nationwide study from 1955 through 2013. Pituitary 18(6):803-807.
10. Burton T, Le Nestour E, Neary M, Ludlam WH (2016) Incidence and prevalence of acromegaly in a large US health plan database. Pituitary 19(3): 262-267.

11. Portocarrero Ortiz L, Vergara Lopez A, Vidrio Velazquez M, Uribe Diaz AM, García Dominguez A, et al. (2016) The Mexican Acromegaly Registry: Clinical and biochemical characteristics at diagnosis and therapeutic outcomes. J Clin Endocrinol Metab 101(11): 3997-4004.

12. Rosario PW, Calsolari MR (2012) Screening for acromegaly by application of a simple questionnaire evaluating the enlargement of extremities in adult patients seen at primary health care units. Pituitary 15(2): 179183.

13. Day PF, Loto MG, Glerean M, Picasso MF, Lovazzano S, et al. (2016) Incidence and prevalence of clinically relevant pituitary adenomas: retrospective cohort study in a Health Management Organization in Buenos Aires, Argentina. Arch Endocrinol Metab 60(6): 554-561.

14. López Gavilanez E, Guerrero Franco K, Solórzano Zambrano N, Navarro Chavez M, Lopez Estrella C, et al. (2016) Epidemiology of acromegaly in Ecuador. Endocrinol Nutr 63(7): 333-338.

15. http://www. ecuadorencifras.gob.ec/censo-de-poblacion-y-vivienda

16. https://sni.gob.ec/proyecciones-y-estudios-demograficos

17. Kwon O, Song YD, Kim SY, Lee EJ (2013) nationwide survey of acromegaly in South Korea. Clin Endocrinol (Oxf) 78(4): 577-585.

18. Bex M, Abs R, T'Sjoen G, Mockel J, Velkeniers B, et al. (2007) AcroBel-the Belgian registry on acromegaly: a survey of the 'real-life' outcome in 418 acromegalic subjects. Eur J Endocrinol 157(4): 399-409.

19. Acosta Naranjo WD, Salazar Vega JL, Carrillo Rivadeneira DP (2018) Características clínicas de los pacientes con acromegalia atendidos en el Servicio de Endocrinología del Hospital Eugenio Espejo, Quito, Ecuador. Revista Colombiana de Endocrinología, Diabetes y Metabolismo 5(2):84. available in http://revistaendocrino.org/ accessed May 20, 2020

20. Broder MS, Chang E, Cherepanov D, Neary MP, Ludlam WH, et al. (2016) Incidence and prevalence of acromegaly in the united states: a claimsbased analysis. Endocr Pract 22(11): 1327-1335. 